Meta

Journal des traducteurs

Translators' Journal

\title{
Les industries de la langue et la traduction en Belgique francophone
}

\section{Thierry van Steenberghe}

Volume 39, numéro 1, mars 1994

La traduction et l'interprétation dans la Belgique multilingue

URI : https://id.erudit.org/iderudit/004293ar

DOI : https://doi.org/10.7202/004293ar

Aller au sommaire du numéro

Éditeur(s)

Les Presses de l'Université de Montréal

ISSN

0026-0452 (imprimé)

1492-1421 (numérique)

Découvrir la revue

Citer cet article

van Steenberghe, T. (1994). Les industries de la langue et la traduction en Belgique francophone. Meta, 39(1), 132-149. https://doi.org/10.7202/004293ar
Résumé de l'article

L'article décrit la situation des industries de la langue en Belgique francophone, considérées du point de vue de la traduction. La diffusion des produits et des services pour la Traduction Automatique ou Assistée par Ordinateur (TA ITAO) et pour les Outils d'Aide à la Traduction (OAT) est passée en revue, et les pratiques et attitudes vis-à-vis de ces nouvelles technologies dans les divers segments de la profession de traducteur sont examinées. Les informations ont été en partie collectées à l'occasion d'une étude de l'état des industries de la langue pour la traduction, faite en 1992 à la demande du Ministère de la recherche et des nouvelles technologies de la Région wallonne de Belgique. 


\title{
LES INDUSTRIES DE LA LANGUE ET LA TRADUCTION EN BELGIQUE FRANCOPHONE
}

\author{
THIERRY VAN STEENBERGHE \\ Université Catholique de Louvain, Institut de Linguistique, Louvain-la-Neuve, Belgique
}

\begin{abstract}
Résumé
L'article décrit la situation des industries de la langue en Belgique francophone, considérées du point de vue de la traduction.

La diffusion des produits et des services pour la Traduction Automatique ou Assistée par Ordinateur (TA/TAO) et pour les Outils d'Aide à la Traduction (OAT) est passée en revue, et les pratiques et attitudes vis-à-vis de ces nouvelles technologies dans les divers segments de la profession de traducteur sont examinées.

Les informations ont été en partie collectées à l'occasion d'une étude de l'état des industries de la langue pour la traduction, faite en 1992 à la demande du Ministère de la recherche et des nouvelles technologies de la Région wallonne de Belgique.
\end{abstract}

\section{INTRODUCTION}

\section{La Belgique et la traduction}

La Belgique est, par tradition, une terre de multilinguisme. Ces territoires plats et fertiles ont attiré au cours des âges de nombreux conquérants, guerriers ou marchands, qui apportaient avec eux leurs cultures et leur langues: Normands, Romains, Germains, puis Italiens, Espagnols, Autrichiens, Bourguignons et Français, Hollandais, et bien d'autres encore.

Tant d'aventures laissent des traces: la Belgique d'aujourd'hui parle trois langues: le néerlandais, le français et l'allemand. Ceci ne simplifie pas la vie politique du pays, mais offre tout de même des avantages: ce pays est très ouvert aux langues, et la traduction y est donc tout naturellement devenue une spécialité.

La Belgique moderne continue d'ailleurs d'être - dans une certaine mesure - une terre d'accueil : des groupes divers y ont fait souche, et ont encore apporté de nouvelles cultures et de nouvelles langues. C'est ainsi que l'on trouve, surtout à Bruxelles et dans les régions industrielles de Wallonie, d'importantes et parfois déjà anciennes communautés italienne, marocaine, et turque, pour ne citer que les principales.

De plus, le pays a attiré nombre d'entreprises multinationales ainsi que quelques grands organismes internationaux, dont la Commission des Communautés européennes avec (aujourd'hui) neuf langues de travail. Ceci a sans doute encore accru la conscience du multilinguisme.

Il n'est donc pas étonnant que la Belgique forme tant de traducteurs et d'interprètes, dans une série d'institutions de niveau universitaire.

Comment ces traducteurs et ces traductrices vivent-ils les transformations que l'informatisation a introduites et continue d'introduire dans leur métier?

Que font ces nouvelles industries de la langue pour le traducteur?

Comment peut-on aussi favoriser une utilisation optimale des nouveaux produits que ces industries proposent? 
Comment encourager enfin l'innovation dans ce domaine, dynamiser ces nouvelles industries et les inciter à préciser leur offre?

Ce sont là quelques questions auxquelles devait répondre une enquête sur les industries de la langue demandée par le Ministre de la recherche, des technologies et des relations extérieures de la Région wallonne de Belgique, en support de sa politique scientifique et technique.

L'ensemble de cette étude a été présenté et discuté lors du Forum des industries de la langue tenu à Bruxelles en mai 1993 (OWIL 1993).

Le présent article propose un tour d'horizon du paysage de l'informatisation de la traduction en Belgique francophone, tel qu'on peut le voir aujourd'hui, en s'appuyant sur les résultats obtenus au cours de la partie de cette étude consacrée à la traduction, menée en 1992 (van Steenberghe 1992).

\section{LES INDUSTRIES DE LA LANGUE}

Les industries de la langue (en abrégé IL) recouvrent un large domaine aux frontières floues. Cette appellation désigne en effet toutes les activités liées à l'utilisation des technologies informatiques pour le traitement des informations en langue naturelle. Une présentation de ce que recouvre cette expression est donnée dans (OCIL et OWIL 1991), ou plus en détail dans (Corbeil 1990).

Les principaux secteurs généralement distingués dans le domaine sont les suivants :

- le traitement de la parole;

- l'informatique documentaire;

- la bureautique avancée;

- la traduction automatique et assistée par ordinateur.

Des points de contact et des recouvrements apparaissent souvent entre ces secteurs : le secteur traduction a des connexions étroites avec chacun des autres secteurs, et particulièrement avec le secteur bureautique.

\section{QUELS PRODUITS IL POUR LA TRADUCTION?}

Un critère utilisé pour catégoriser les produits IL pour le secteur de la traduction correspond à une dimension de granularité qui permet de classer les systèmes en trois grands groupes :

- traduction automatique (TA);

- traduction assistée par ordinateur (TAO);

- outils d'aide à la traduction (OAT).

Cette division commode permet de représenter le degré d'automaticité des produits proposés, leur richesse fonctionnelle, leur complexité, leur puissance, et... leur prix.

La même dimension permet aussi de classer les types d'utilisation, et, par voie de conséquence, les types d'utilisateurs de ces produits : les caractéristiques pertinentes sont ici le volume des traductions et la qualité qui leur est demandée, souvent liées à l'environnement socio-organisationnel.

Cette dimension correspond enfin, par une relation de proportionnalité inverse, à la diffusion actuelle et potentielle des produits sur le marché, puisque la complexité et l'ampleur de l'investissement requis pour les systèmes les plus puissants freinent leur diffusion. 
La traduction assistée par ordinateur (en abrégé TAO) recouvre tout ce que l'on nomme parfois de façon générique Traduction Automatique (ou MT, pour Machine Translation).

La TAO ou Traductique peut aussi être entendue au sens le plus large comme comprenant tous les produits et toutes les méthodes destinées à assister le traducteur humain dans son travail: on parle parfois de traduction humaine assistée par ordinateur ou THAO.

\section{TRADUCTION AUTOMATIQUE, TRADUCTION ASSISTÉE ?}

La traduction automatique (en abrégé TA) a été envisagée dès l'apparition des premiers ordinateurs, à la fin de la Deuxième Guerre mondiale.

Après les premières désillusions, des résultats très encourageants ont été obtenus par les équipes de R\&D actives un peu partout dans le monde, au point qu'on voit apparaître aujourd'hui des systèmes commerciaux ou quasi commerciaux, en dépit des énormes difficultés rencontrées.

Ces difficultés tiennent au fait que le sens dans le langage naturel est très difficile à formaliser. Traduire consiste en effet à comprendre un texte dans une langue pour le réécrire ensuite dans une autre.

Aujourd'hui, les méthodes de représentation de la signification sont donc au cœur du problème, surtout si l'on veut comprendre la TA comme une traduction de haute qualité complètement automatique (FAHQT, Fully Automatic High Quality Translation).

C'est encore là un domaine de recherche fondamentale.

\section{la Ta / TAO AUJOURd'hui, POUR QUi, POUR QUOI?}

Si la traduction complètement automatique et de haute qualité de n'importe quel texte n'est pas pour demain, à quoi peut donc servir un système de traduction semi automatique et de qualité... moins grande ?

Les éléments de réponse à cette question tiennent à la fois aux types de textes et de demandes pour les traductions.

Les textes littéraires au sens large (poésie, littérature, publicité, etc.), ne se prêtent pas à la traduction automatique; ils ne représentent d'ailleurs qu'une très faible partie du volume traduit total.

Il reste un volume énorme de documents de toutes sortes (scientifiques et techniques, économiques, sociaux, administratifs, etc.) qui devraient être traduits. Tous ne peuvent l'être, en raison du manque de traducteurs, du volume, du temps et des coûts, mais la traduction peut être modulée en fonction des besoins. Ainsi, pour des activités de veille scientifique ou économique, une traduction brute peut suffire, et c'est là un créneau privilégié pour la traduction automatique.

Par ailleurs, si l'on a fréquemment à traduire de grands volumes de textes de même type, assez standardisé, comme des textes administratifs, voire répétitifs, comme des rapports ou des manuels, la machine peut se charger avec efficacité de cette tâche peu exaltante pour un traducteur humain et ne laisser à celui-ci que la révision à effectuer.

On peut donc penser que la TA n'est pas d'abord destinée aux traducteurs, mais plutôt aux professionnels en quête d'information qui ne peuvent lire les textes originaux mais se satisfont d'une traduction brute, ou aux organismes qui souhaitent automatiser leur production de documents multilingues.

Par contre, la traduction assistée et les outils d'aide à la traduction peuvent dès aujourd'hui apporter une aide appréciable au traducteur professionnel ou occasionnel. 


\section{DE LA TA À LA TAO, ET DE LA TAO AUX OAT}

\section{Traduction automatique (TA)}

Il s'agit de systèmes permettant la traduction d'un texte par la machine seule, sans intervention notable de personnel qualifié en traduction pendant le processus de traduction proprement dit.

Des interventions de pré-édition sont cependant généralement nécessaires pour préparer un travail efficace, et une post-édition est indispensable si le résultat doit être parachevé.

La traduction est automatique, au sens où elle est réalisée par la machine seule.

\section{Traduction assistée par ordinateur (TAO)}

En traduction assistée, ou interactive, le traitement est lancé, après une éventuelle pré-édition, par un opérateur qui doit ensuite intervenir lorsque la machine l'y invite, par exemple pour la désambiguïsation, le choix lexical, etc. Le traitement est alors interrompu et relancé lors de l'interaction avec la machine. En plus de la connaissance du système qu'il pilote, l'opérateur doit avoir dans ce cas des qualifications pour la paire de langues concernées.

\section{Outils d'aide à la traduction (OAT)}

Il y a un degré d'arbitraire à tenter de fixer une limite entre la TAO et les OAT: il existe une solution de continuité qui mène des petits systèmes TAO à de gros systèmes OAT.

On parlera de préférence d'OAT lorsqu'il s'agit de systèmes ou de sous-systèmes plus ou moins intégrés, mis à la disposition du traducteur pour l'aider dans son travail, qualitativement ou /et quantitativement.

\section{QUI SONT LES UTILISATEURS DES PRODUITS IL POUR LA TRADUCTION?}

On peut tenter de distinguer, parmi les utilisateurs actuels ou potentiels des produits IL pour la traduction, les traducteurs professionnels, les professionnels nécessitant occasionnellement des traductions pour leurs besoins propres ou devant rédiger en langue non maternelle, et enfin le grand public.

La catégorie des traducteurs professionnels compte, en Belgique, plus de 2500 personnes, auxquelles s'ajoutent les nombreux traducteurs des grandes organisations internationales installées dans le pays, comme la Commission des Communautés Européennes (CCE) ou l'OTAN.

Il est sans doute intéressant de noter que, outre les associations d'écoles, deux associations professionnelles s'efforcent de regrouper les traducteurs: quelque $10 \%$ des traducteurs seulement se rattachent à l'une ou l'autre de ces associations.

Il faut observer que les traducteurs professionnels forment un groupe très hétérogène, leurs environnements professionnels conditionnant de façon déterminante leurs besoins, leurs pratiques, et leurs attitudes. On peut ainsi distinguer, en première approximation, le traducteur indépendant, le traducteur de bureau, travaillant dans un bureau de traduction, et le traducteur interne, employé par un organisme dont le personnel est son seul client.

Les systèmes de TA/TAO les plus puissants ne peuvent sans doute intéresser que des organismes ayant à traduire (avec ou sans révision) des volumes importants, ou de grands bureaux, alors que les organismes ayant des volumes moindres à traduire, les petits bureaux et les traducteurs indépendants seront peut-être davantage intéressés par les stations de TAO et sûrement par les OAT. 
Les professionnels non traducteurs seront, quant à eux, essentiellement concernés par les OAT, ou par la TA pour les activités de veille.

\section{TRADUCTION AUTOMATIQUE ET TRADUCTION ASSISTÉE PAR ORDINATEUR}

L'histoire de la TA/TAO a déjà été relatée (Hutchins 1986), et il n'est donc pas nécessaire de la reprendre ici.

Depuis le début des années 70, nombre de nouveaux projets ont été lancés en Amérique du Nord, en Europe, et au Japon. On pourra en trouver une revue dans les récents rapports (Varile et Zampolli 1992), (JEIDA 1992) et études de marché (Ovum 1991) consacrés à la TA.

Pour ce qui est de la qualité de traduction, le premier résultat des systèmes de TA est aujourd'hui au mieux une traduction brute, où les ambiguïtés lexicales et sémantiques ne peuvent encore être complètement résolues par la machine et doivent donc être éliminées par un travail soigné de post-édition.

Cette caractéristique contribue largement à expliquer la méfiance vis-à-vis des systèmes de $T A$, du moins chez ceux qui en attendraient une traduction du même niveau que celle que produisent des traducteurs professionnels, ou chez ceux qui y sont hostiles par principe.

Même dans le cas de domaines restreints de connaissance et de types de textes bien définis, il est nécessaire d'ajuster tout système de TA/TAO à son utilisation pendant une période de rodage suffisante si l'on veut obtenir un outil efficace.

Le plus grand défi pour l'ensemble des systèmes TA/TAO développés jusqu'ici est manifestement la prise en compte des niveaux sémantiques et pragmatiques profonds. Ceci est universellement reconnu, même si les méthodes qui devraient permettre de résoudre ce problème sont loin de faire l'unanimité (COLING 1992; TMI 1992; Hutchins et Somers 1992).

L'application de méthodes et de techniques produites par les progrès en représentation des connaissances obtenues par la recherche en intelligence artificielle est une des voies les plus prometteuses: on parle de TA à base de connaissances (KBMT, pour Knowledge Based Machine Translation) pour les systèmes qui appliquent de telles méthodes à la TA, par exemple en utilisant le raisonnement sur la signification du texte à traduire (Fargues et Sabah 1992), (Nirenburg et al. 1992).

Des recherches dans ce domaine sont aussi menées en Belgique.

Un nouveau paradigme de la TA est apparu récemment: la TA basée sur les exemples, (EBMT, pour Example Based Machine Translation), quelquefois appelée aussi TA basée sur la mémoire (MBMT, pour Memory Based MT), initialement proposée par Nagao (Nagao 1984).

Les traducteurs utilisent souvent ce modèle de façon empirique, en faisant appel à leur propre mémoire ou à un système de classement qui doit leur permettre de retrouver rapidement le texte d'une version antérieure, ou similaire à celui qu'ils ont à traduire.

\section{LES GRANDS SYSTÈMES COMMERCIAUX DE TA/TAO, VUS DE BELGIQUE}

Les systèmes les plus connus aujourd'hui en Europe, et en Belgique en particulier, sont le système SYSTRAN et le système METAL de Siemens-Nixdorf.

Le système LOGOS, assez répandu en Europe, n'est guère présent en Belgique. Il est amusant de noter au passage qu'un de ses grands clients européen était... Nixdorf, aujourd'hui fusionnée à Siemens.

SYSTRAN est le plus ancien des systèmes de TA, et celui qui offre le plus grand nombre de paires de langues. 
Il a été adopté quelque temps par un grand bureau de traduction bruxellois, mais ce n'est plus le cas, essentiellement pour des raisons d'ordre commercial.

SYSTRAN a été acquis par la Commission des Communautés Européennes (CCE) dans les années 70 pour utilisation interne. Il y est toujours utilisé, et reçoit même une certaine promotion puisqu'il est offert aujourd'hui gratuitement et en libre-service par le service de traduction de la CCE à tous les fonctionnaires disposant de l'accès au réseau informatique interne, pour les traductions brutes. Ces traductions peuvent être faites au départ de l'anglais, du français, de l'allemand, et de l'espagnol : les meilleurs résultats sont annoncés dans les paires français-anglais (la plus demandée), anglais-français, françaisitalien et anglais-italien (CCE-SYSTRAN).

Le système est très rapide: les textes courts sont en principe traduits et renvoyés par courrier électronique dans l'heure qui suit la demande. Une enquête menée auprès des utilisateurs par l'Université d'Essex devrait renseigner sur leur opinion.

Le développement continue à Luxembourg, où l'ordinateur hôte est situé.

Il est peut-être significatif de noter ici que ce service de traduction (un des plus importants au monde : 1500 personnes - à comparer aux 800 qu'emploie l'ONU —, et 1000000 pages traduites annuellement), établi à Bruxelles et à Luxembourg, propose le service SYSTRAN aux autres fonctionnaires, mais ne l'utilise pas lui-même ou peu.

Une partie des droits commerciaux sur SYSTRAN ont été rachetés en 1982 par le Groupe Gachot qui a aussi acquis la plupart sinon toutes les compagnies Systran locales: ce groupe contrôle donc le développement (hors $\mathrm{CCE}$ ) du système. Il a aussi des accords avec Xerox qui offre cependant son système GlobalView/DocuTran.

Le Groupe Gachot propose SYSTRAN non seulement comme produit à utiliser sur l'ordinateur du client, mais aussi comme service accessible à partir d'un micro-ordinateur distant, avec la possibilité de construire et d'entretenir une base de données terminologiques propre. Une version limitée a même été rendue accessible au public sur le Minitel, en France, et donc en Belgique par l'entremise de la passerelle du Vidéotex. Ces possibilités ne semblent pas rencontrer un grand succès en Belgique.

Le système METAL a lui aussi été initialement développé aux États-Unis (University of Texas, Austin) depuis la fin des années 50.

Siemens-Nixdorf Informationssysteme AG (SNI) s'y est intéressée dès 1978 pour ses besoins internes: la section Développement de produits en a repris le développement depuis 1985, et le commercialise depuis 1988 (Schneider 1992; Benett 1991; SNI). Une vingtaine de clients, dont des industriels, des banques, des institutions publiques ou d'enseignement, et de grands bureaux de traduction utilisent aujourd'hui METAL, en Allemagne et en Suisse, surtout. C'est actuellement le seul système de TA adopté par une grande compagnie multinationale.

Â la fin de 1985, le système METAL a été transféré en Belgique pour le développement, cofinancé par Siemens et l'État belge (IRSIA), des paires néerlandais-français et français-néerlandais par un groupe de chercheurs de l'Université de Louvain (KUL) à Leuven et de l'Université de Mons. Ce groupe est maintenant installé au Centre Software de Liège (CSL) de Siemens, où des chercheurs de l'Université de Liège ont collaboré au développement des paires français-anglais.

Les deux paires néerlandais-français et français-néerlandais sont commercialement disponibles. Un système comportant ces deux paires (soit un couple, dans la terminologie Siemens) est utilisé pour les besoins internes de Siemens-Nixdorf à Bruxelles, et un autre est aujourd'hui exploité en production au Ministère de l'Intérieur, à Bruxelles, où son développement a été parachevé pour les textes administratifs et légaux avec la collaboration d'agents du service de traduction du ministère, en particulier pour les lexiques terminologiques. 
METAL offre ou annonce encore plusieurs autres combinaisons de langues, dont les paires français-anglais, français-allemand, et le développement se poursuit entre autres à Liège.

Le système, initialement basé sur une machine LISP Symbolics 36x0 avec une frontale desservant des terminaux, peut travailler pour plusieurs utilisateurs simultanés. Un tel équipement peut supporter trois ou quatre paires de langues.

Une version fonctionnant sur station de travail SUN est apparue récemment et ceci devrait permettre une plus large diffusion du produit.

La productivité moyenne d'un traducteur professionnel varie fort avec la difficulté des textes: pour des textes techniques on l'estime généralement à un nombre entre 5 et 10 pages par jour. L'expérience au Ministère de l'Intérieur montre qu'un système METAL avec un seul traducteur peut traduire, pré-édition et post-édition comprises, quelque 30 pages par jour, après la période d'apprentissage et d'ajustement du système. Le système étant prévu pour desservir plusieurs traducteurs, et pouvant bien sûr traiter la nuit des textes préédités pendant le jour, on imagine l'augmentation de production possible, d'autant que la qualité des résultats semble très satisfaisante.

La commercialisation de METAL français-néerlandais ne fait que commencer, mais le prix du système semble cependant faire obstacle à sa diffusion: cet aspect des choses devrait s'améliorer avec la version sur station SUN.

\section{DES SYSTÈMES DE TAO SUR MICRO-ORDINATEURS ?}

Certains systèmes de TAO sur micro-ordinateurs, comme PC-Translator ou GTS de Globalink (distribué en Belgique, où une vingtaine d'exemplaires seraient utilisés), peuvent se révéler très performants entre les mains d'un utilisateur competent qui a pu prendre le temps de s'initier sérieusement au système et de l'ajuster à ses besoins.

Ces deux produits offrent, parmi d'autres, les paires français-anglais.

D'après des utilisateurs qui ont comparé de tels systèmes TAO sur micro-ordinateur avec des systèmes sophistiqués comme ceux décrits ci-dessus, la qualité moindre de la traduction brute serait plus que compensée par la vitesse de travail, surtout si l'on tient compte des prix relatifs.

D'autres produits pour micro-ordinateurs, moins ambitieux, les Language Assistant de MicroTac, proposés à prix modestes pour la traduction individuelle de l'anglais vers diverses langues, dont le français, donnent des résultats... tout aussi modestes. De grands progrès ont été faits, semble-t-il, dans les demières versions, devenues bidirectionnelles, et qui donnent de bien meilleurs résultats. Hélas, en Belgique comme en France, c'est encore l'ancienne version, unidirectionnelle de l'anglais vers le français, qui est commercialisée par Softissimo.

Par ailleurs, on observe avec intérêt les annonces de portage de versions allégées des grands systèmes de TAO vers les micro-ordinateurs.

Aujourd'hui, aucun système sur micro-ordinateur n'offre encore les paires nationales français-néerlandais, en raison sans doute de l'étroitesse relative du marché; si c'était le cas, on peut imaginer que la diffusion d'un tel produit serait importante en Belgique.

\section{LES OUTILS D'AIDE À LA TRADUCTION}

\section{Équipement informatique de base}

La révolution micro-informatique a bien sûr profondément modifié les pratiques des traducteurs, en Belgique comme ailleurs.

L'enquête (Guérain 1990) annonçait que $60 \%$ des traducteurs en Belgique travaillaient sur ordinateur : ce taux était sans doute sous-estimé, étant donné les caractéristiques 
de l'échantillon. Il est aujourd'hui impensable de pratiquer encore la traduction professionnelle sans micro-ordinateur: à l'engagement d'un traducteur, on ne lui demande plus si un traitement de texte lui est connu, mais bien lequel...

Ces ordinateurs sont en grande majorité (au moins $80 \%$ ) de type PC-compatible sous DOS (et parfois Windows), quelquefois raccordés en réseaux, le solde se répartissant entre les machines Macintosh, les stations de travail sous UNIX et des terminaux raccordés à diverses machines centrales.

Un aspect important, mais souvent oublié, de l'informatisation de la traduction, et plus généralement du multilinguisme, est celui des caractères non latins, des diacritiques spécifiques à des langues moins courantes, et aussi des symboles mathématiques et scientifiques ou techniques.

Le problème se pose pour les claviers, les écrans et les imprimantes. Il y a là un marché, étroit peut-être, mais néanmoins prometteur. Quelques petites entreprises belges se sont positionnées dans ce créneau, comme Irisoft à Bruxelles.

\section{Traitement de Texte}

L'orientation multilingue se généralise dans les logiciels de traitement de textes, soit par l'ajout de modules de langue, soit par l'introduction de nouvelles versions ou de nouveaux logiciels spécialement conçus dans cette perspective, éventuellement à la faveur de la diffusion croissante des interfaces graphiques.

Le traitement de texte le plus répandu en Belgique (au moins $2 / 3$ des traducteurs, d'après Guérain 1990), comme dans le monde, est WordPerfect. Les modules de langue (correcteurs orthographiques et dictionnaires de synonymes) sont disponibles pour une vingtaine de langues, dont bien sûr nos langues nationales. Un autre aspect apprécié est la simplicité du transfert des fichiers pour la plupart des plates-formes.

Il existe pour les traitements de texte les plus répandus des répertoires de macrocommandes prêtes à l'emploi pour de nombreuses tâches générales, mais, à notre connaissance, aucun spécifiquement conçu pour les traducteurs: ils doivent donc les écrire eux-mêmes, ou les obtenir de collègues.

Les logiciels de traitement de texte montrent aujourd'hui une évolution vers la PAO (publication assistée par ordinateur), liée aussi à la diffusion des interfaces graphiques. Les logiciels PAO proprement dits sont parfois utilisés par les traducteurs, dans la mesure où ils ont à produire des travaux proches du stade final, et en particulier des documents incorporant des éléments graphiques, ou des documents multilingues avec une présentation commune. C'est rarement le cas pour les traducteurs indépendants, mais plus souvent pour les bureaux qui offrent un service complet, et où la partie PAO peut être laissée à des spécialistes.

La pratique courante en Belgique est encore que les textes soient remis au traducteur uniquement sous forme de document imprimé sur papier. Il est par contre presque toujours demandé au traducteur de fournir la traduction à la fois imprimée sur papier et sous forme électronique, sur disquette, le plus souvent.

Il est en tout cas très rare qu'un traducteur indépendant reçoive les textes à traduire sous forme électronique. Ceci n'encourage pas l'utilisation de systèmes de TAO, sauf à disposer d'un scanner avec logiciel d'OCR. Il faut mentionner ici l'entreprise IRIS de Louvain-la-Neuve, qui a réalisé un excellent logiciel OCR (ReadIRIS) avec un impressionnant support multilingue.

\section{Dictionnaires électroniques}

L'évolution très rapide des moyens (magnétiques ou optiques) de stockage de l'information, à la fois en termes de coût et en termes de capacité, a conduit entre autres à l'apparition de dictionnaires électroniques. 
Comme leurs équivalents sur papier, ces dictionnaires sont de différents types: dictionnaires de langue et de synonymes monolingues, bilingues ou multilingues, dictionnaires spécialisés, encyclopédies, bases de données terminologiques, etc.

Le support privilégié est aujourd'hui le CD-ROM. On doit citer ici un produit phare en langue française : Le Grand Robert, mis au point sur la base des fichiers de la version papier de l'éditeur français par le Bureau Marcel Van Dijck, à Bruxelles. Des produits semblables existent en néerlandais (Van Dale, Lexitron) et en anglais (OED, Webster, Collins).

Ces dictionnaires, de même que les encyclopédies électroniques sur CD-ROM, telle que Zyzomis de Hachette, en français, ou les encyclopédies américaines, sont cependant encore peu utilisés par les traducteurs en Belgique : le coût de ces produits et des lecteurs nécessaires les réservent en effet aux grands bureaux, qui disposent souvent déjà de la version papier...

Le support magnétique offre aussi, malgré sa capacité plus réduite, des produits intéressants et toujours plus accessibles. Ces produits, pour la plupart nouveaux, sont souvent peu connus et ne sont donc guère utilisés en Belgique.

De même pour les bilingues, les versions électroniques de produits déjà connus sur papier, comme les dictionnaires traductifs Collins, fonctionnant avec la plupart des traitements de texte ou indépendamment, sont peu utilisés en Belgique: l'absence de bilingue français-néerlandais en est sans doute à nouveau l'une des raisons.

Cependant, des produits plus originaux, comme l'Euroglot de la néerlandaise Linguistic Systems ou le Lexica de Wordstar, à la fois dictionnaires traductifs et dictionnaires de synonymes multilingues comportent à présent nos langues nationales.

Enfin, on rencontre encore très peu les dictionnaires spécialisés en version électronique, comme les dictionnaires terminologiques d'Elsevier/Eurolux (aux formats MTX ou MultiTerm), ou, dans un registre plus général, les Bilingues de Larousse (affaires, informatique).

\section{Bases de données terminologiques}

La terminologie est bien sûr cruciale en traduction spécialisée, qu'il s'agisse de sciences, de technologie, d'économie, de législation, ou de tout autre domaine où un sous-langage précis a été développé.

On a vu apparaitre ces dernières années des produits et des services informatiques pour la gestion terminologique: bases de données bilingues ou multilingues, fournies sur CD-ROM (avec mises à jour régulières), comme la base canadienne TERMIUM, ou installées sur un ordinateur hôte accessible par ligne téléphonique par l'entremise d'un modem, comme la base multilingue EURODICAUTOM de la CCE (Reichling 1992), située à Luxembourg et facilement accessible depuis la Belgique (en attendant son transfert sur CD-ROM).

En Belgique même, le Ministère des Affaires Économiques a récemment annoncé l'accès à une base terminologique multilingue BELGOTERM, provisoirement centrée sur les industries de pointe, informatique, télécommunications, robotique, avec aussi de la terminologie statistique et économique générale, et s'étendant progressivement à d'autres domaines techniques. Cette base sera accessible en ligne, et on envisage d'en réaliser une version sur CD-ROM.

Le problème de la terminologie reste pourtant toujours complexe en raison des nombreux sous-langages spécialisés: les termes techniques les plus précis sont très souvent l'objet d'un emploi différencié d'un secteur à l'autre, ou même souvent déterminés par une culture d'entreprise spécifique.

La gestion des glossaires est par conséquent une activité quotidienne du traducteur. Cette tâche, traditionnellement conduite à partir de fiches reprenant un ensemble d'infor- 
mations utiles à la sélection du terme recherché, se prête particulièrement bien à l'informatisation. Il n'est cependant pas rare de trouver encore, et même dans de grands services de traduction, d'archaïques listes crayonnées sur papier ou des fiches en carton, rangées dans un tiroir, si ce n'est dans la classique boîte à chaussures, et souvent emportées par leur auteur lors de son départ.

Les traducteurs ont donc besoin de produits qui leur permettent de constituer euxmêmes leurs propres fichiers terminologiques et de les exploiter ensuite efficacement. Les traducteurs informatisés ont vite perçu les possibilités de leur ordinateur pour la terminologie, et les plus entreprenants ont développé eux-mêmes des fichiers électroniques: cela va de la simple liste de termes en traitement de texte jusqu'à l'utilisation des systèmes de gestion de bases de données génériques, en passant par des systèmes spécifiques créés de toutes pièces par un traducteur pour les besoins de son service, le plus souvent durant ses loisirs!

Des produits spécifiques standardisés, plus simples d'emploi que les classiques systèmes de gestion de base de données à tout faire, sont cependant proposés: les plus connus sont les systèmes Mercury/Termex (ou MTX) de LinguaTech, et MultiTerm de INK/Trados, mais ces produits sont assez peu utilisés en Belgique.

La mise en réseau des machines permet aussi de partager une base terminologique commune dans une organisation où plusieurs traducteurs travaillent sur les mêmes types de texte, et peuvent alors non seulement consulter la base commune, mais aussi contribuer à son enrichissement continu. Du point de vue de l'organisation, une telle méthode offre bien des avantages : cohérence, richesse, rapidité, permanence. Elle est cependant encore rare en Belgique: même lorsqu'un réseau existe, la terminologie est souvent laissée à l'initiative individuelle.

À noter encore qu'il existe en Belgique francophone plusieurs centres de terminologie, dont les activités sont orientées vers le développement de logiciels ou vers la production de bases terminologiques, comme le Centre de terminologie de l'Institut Marie-Haps, ou l'ISTI, à Bruxelles.

\section{Correcteurs orthographiques, grammaticaux, stylistiques}

Les correcteurs orthographiques intégrés ou associés aux logiciels de traitement de texte sont utiles pour s'assurer de l'orthographe, mais aussi pour repérer les fautes de frappe. Celles-ci ne perturbent guère la lecture du traducteur, mais bien celle d'un système de TAO: il est donc indispensable qu'une vérification orthographique soit faite avant toute traduction automatique, car un mot mal écrit est un mot inconnu, ou pire, un contre-sens.

Les correcteurs syntaxiques et stylistiques et les conjugueurs, apparus depuis quelques années, offrent de nouvelles possibilités de vérification.

On peut cependant se demander, au moins dans l'état actuel du développement de ces produits, si ces outils sont bien utiles pour la langue maternelle?

De tels produits existent pour plusieurs langues, dont le français, mais pas encore pour le néerlandais.

À signaler que le correcteur Grammatik pour le français, et aussi la version pour l'anglais pour francophones, ont été développés par la filiale belge de Reference Software, à Anvers, qui en prépare une version néerlandaise. Cette société a aujourd'hui été reprise par WordPerfect qui inclut Grammatik dans les nouvelles versions de son traitement de texte.

Un autre outil de ce type est constitué par les grammaires en ligne. Il s'agit ici plutôt d'ouvrages de référence sous forme électronique permettant au rédacteur ou au traducteur de lever un doute ou de s'autocorriger.

La Belgique a une longue tradition de grammairiens et a donc apporté sa contribution: ainsi, en français, Le Grammairien, de l'éditeur Softissimo, est basé sur la célèbre grammaire 
Grevisse/Goosse; en anglais, on peut citer English Grammar de la société Didacta, à Bruxelles.

Les correcteurs peuvent être utiles à la révision, mais aussi pour une sorte de contrôle de qualité des textes sources: la mauvaise rédaction des textes remis pour traduction est en effet à l'origine de bien des difficultés. Ce contrôle devrait bien sûr être une routine du rédacteur, mais le traducteur peut préférer s'en assurer, surtout s'il compte faire usage d'un système de TAO.

\section{Utilitaires}

L'échange de documents sous forme électronique est encore trop souvent rendu difficile par l'incompatibilité des formats de fichiers. En attendant une éventuelle normalisation, par exemple au format SGML, il existe pour résoudre ce problème des utilitaires de conversion efficaces, mais pas toujours parfaits, intégrés dans les traitement de texte ou indépendants.

Un problème associé est celui de la conservation des codes de formatage et de mise en page de documents lorsqu'ils sont disponibles sous forme électronique: on souhaite en général voir la traduction présentée sous une forme aussi proche que possible de celle du texte de départ. Des logiciels particuliers permettent de filtrer les codes de mise en page dans le texte source, et de les réintroduire ensuite dans la traduction. Un tel produit est le langage Adapt développé par la société belgo-canadienne Destin, à Nivelles, qui offre aujourd'hui ce travail spécialisé en tant que service.

Les télécommunications entre ordinateurs et le courrier électronique n'ont pas encore vraiment conquis les utilisateurs en Belgique. Les traducteurs indépendants travaillant pour des bureaux sont souvent équipés de modems et font ici figure de pionniers.

L'usage de la télécopie est par contre très (trop?) répandu chez les traducteurs, comme dans tous les secteurs professionnels: la facilité d'emploi en compense l'inefficacité. Moyen terme, la carte fax pour micro-ordinateur se généralise.

Enfin, on ne peut ignorer les problèmes pratiques de gestion et d'organisation : remises d'offres, facturation (comptage des lignes ou des mots traduits), gestion des adresses et des documents.

Le produit TextCount est souvent considéré comme la référence pour le comptage des lignes traduites, unité habituelle de facturation en Belgique. Pour les adresses, chacun constitue son fichier à sa façon: cela va du paquet de fiches jusqu'à la base de données relationnelle. La gestion électronique des documents (électroniques ou non) est encore nouvelle et donc souvent inconnue.

\section{Les systèmes d'archivage et d'alignement}

La retraduction est un phénomène courant, en raison notamment de la difficulté à retrouver un texte déjà traduit: récupérer ce texte peut en effet prendre plus de temps que le retraduire, si l'on ne dispose pas d'un système d'archivage efficace.

Un tel système d'archivage doit permettre de conserver sur support magnétique ou optique les textes déjà traduits, et de les retrouver ensuite grâce à une indexation ou à un système de recherche full text.

On trouve en Belgique divers systèmes de base de données textuelles, comme Seconde, de la belgo-canadienne Destin, déjà citée. Un système générique d'archivage et d'indexation est aussi fourni par WordPerfect avec les nouvelles versions de son traitement de texte.

Une étape importante du processus de traduction, qu'elle soit d'ailleurs manuelle ou automatique, est la révision: elle est d'habitude très peu prisée des traducteurs, en dépit du fait qu'elle requiert un professionnel expérimenté et correspond donc à une valo- 
risation personnelle. Il n'existe guère de produits spécifiquement destinés à cette activité, si l'on excepte une annonce récente de Trados.

L'alignement est une méthode qui facilite grandement la révision en faisant correspondre des fragments du texte source avec les fragments correspondants du texte cible. L'alignement peut être fait à la main, ce qui représente une tâche énorme s'il n'est pas fait au moment même de la traduction, ou automatiquement grâce à des méthodes développées dans ce but. Des recherches dans ce domaine sont aussi menées en Belgique.

Quelques systèmes d'archivage avec alignement existent sous DOS et sous Windows, mais leur commercialisation est à peine entamée. On peut citer aussi deux produits récents spécifiquement destiné à la traduction, l'un présenté par IBM, le TranslationManager $/ 2$ (sous OS $/ 2$, mais dont on peut attendre une version Windows), et l'autre par Trados, le Translator's Workbench II (sur station Unix).

\section{Un poste de travail du traducteur?}

Il s'agit ici d'un concept de TAO/OAT déjà ancien (Kay 1980, Melby 82), mais dont peu de réalisations sophistiquées ont vu le jour.

L'idée de base consiste à tenter d'intégrer de façon cohérente et efficace sur une station de travail un ensemble d'outils d'aide à la traduction pour permettre les gains de qualité et de productivité rendus possibles par l'informatique (Melby 1992). Ce concept était au centre d'un congrès UREF/AUPELF tenu à l'Université de Mons en 1991 (Clas et Safar 1992).

Une telle station de travail dédiée résulte de l'approche pragmatique développée au Canada, où la loi impose la traduction de la totalité des documents officiels fédéraux. Le poste de travail du traducteur (ou PTT) a ainsi été conçu et réalisé au CCRIT (Centre canadien de recherche sur l'informatisation du travail) (Tremblay 1992). Une deuxième version du PTT a subi une évaluation préalable à sa mise en service (Macklovitch 1992), et une troisième est déjà en chantier.

On peut s'étonner que la Belgique n'ait pas développé ou adopté de système de ce type alors qu'elle est dans une situation de bilinguisme légal du même genre: le journal officiel est bilingue, et le service de traduction du Parlement, où les débats ont lieu dans les deux langues nationales principales, compte environ 80 personnes.

La Commission des Communautés Européennes a, de son côté, lancé, dans le cadre du programme Esprit, un projet de Translator's Workbench ou TWB, similaire au PTT canadien, incluant en outre l'accès à un système de TA/TAO (METAL).

Des systèmes comme TranslationManager/2 d'IBM ou Translator's Workbench II de Trados peuvent aussi être rangés sous l'étiquette de poste de travail du traducteur.

\section{LES PRODUITS TOUS PUBLICS}

Bien qu'il ne s'agisse plus ici de traduction professionnelle, on ne peut ignorer les besoins en multilinguisme des professionnels non traducteurs et du public.

Quelques produits qui peuvent aider un non-traducteur à rédiger dans une langue seconde, ou à faire de courtes traductions pour ses propres besoins ont déjà été cités plus haut : dictionnaires électroniques, correcteurs orthographiques et grammaticaux, conjugueurs, petits systèmes de traduction automatique, éventuellement.

Il s'y ajoute des systèmes d'aide à la rédaction de correspondance en langues étrangères, comme ceux de la société belge Didacta: il s'agit de listes de phrases complètes classifiées pour différentes circonstances répétitives dans la correspondance commerciale, par exemple. De tels produits peuvent être d'une grande utilité lorsqu'on ne peut consulter de locuteurs natifs ou de traducteurs, et que l'on rédige dans une langue qu'on ne maîtrise 
pas parfaitement, mais suffisamment pour pouvoir choisir, et éventuellement modifier, les phrases proposées.

Enfin, on ne peut passer sous silence l'apparition bouillonnante de produits linguistiques comme les traductrices électroniques de poche qui semblent rencontrer un franc succès dans le public: ainsi, l'association belge des consommateurs en offrait comme cadeau de bienvenue à ses nouveaux adhérents!

Cet accueil enthousiaste du public devrait être tout à la fois observé avec prudence et considéré comme un signal positif. $\mathrm{Si}$, en effet, le public semble fasciné par ces produits, c'est qu'il y cherche une réponse à des besoins réels.

Mais la circonspection devrait s'imposer, parce que ce n'est qu'un début de réponse. La déception du public, même due en partie à sa naïveté, pourrait avoir des conséquences négatives, comme la déception des professionnels dans les années 60 a été catastrophique pour la TA : c'est le «syndrome ALPAC».

En tout état de cause, il nous semble que la floraison de produits nouveaux et l'intérêt du public (et aussi la curiosité des professionnels !) sont révélateurs d'un réel besoin, et ceci devrait stimuler le marché.

\section{ÉVALUATION DES PRODUITS}

Il n'y a pas, en Belgique, de centre de compétence en ce qui concerne l'évaluation des systèmes de TA/TAO; au reste, la méthodologie d'évaluation est encore un thème de recherche (King 1992).

Après s'être bien informés, les utilisateurs potentiels doivent procéder à des essais avec des textes typiques, en gardant à l'esprit que les résultats dépendront beaucoup des efforts qu'ils auront à consentir pour l'adaptation et l'amélioration d'un système, au moins pour les lexiques.

Pour l'évaluation des OAT, il n'y a guère d'autres sources que les écrits plus ou moins spécialisés, et les échanges d'informations sur les expériences faites par des collègues ou avec d'autres personnes compétentes.

\section{ENSEIGNEMENT}

La question de savoir quels profils professionnels requièrent les IL pour l'utilisation et le développement de ces produits a fait l'objet de l'une des séances du colloque international sur les IL à Paris en novembre 1991 (TIL-11 1991), et ce thème a été abordé pour la traduction lors des débats d'un colloque organisé par l'EII à Mons en octobre 1992 (Copette et Vande Walle 1993).

Les écoles de traduction ont depuis longtemps pris conscience de la nécessité d'une formation adaptée à l'émergence des nouvelles technologies dans le secteur, et ont ajouté au moins une initiation à l'informatique à leurs programmes : ce mouvement mérite d'être développé. Il est en effet nécessaire de dépasser ce niveau en offrant à des traducteurs la possibilité de se spécialiser dans la connaissance et l'exploitation des produits IL pour la traduction, et en particulier des systèmes de TA/TAO.

Une liste des principaux établissements d'enseignement de Belgique francophone qui proposent un enseignement intéressant le domaine de la traduction est donnée en annexe. Il faut noter que plusieurs de ces institutions éditent des revues, comme Terminologies Nouvelles (RINT/ISTI) ou Le Langage et l' Homme (ILMH).

\section{RECHERCHE ET DÉVELOPPEMENT}

La problématique de la recherche et du développement dans le domaine des IL pour la traduction a fait l'objet d'un numéro antérieur de Meta (Meta 1992) et est par ailleurs 
évoquée du point de vue belge dans le présent numéro: nous ne nous y attarderons donc pas davantage.

L'annexe donne une liste des principaux établissements de Belgique francophone où sont menées des activités de $R \& D$ dans des domaines intéressant la traduction et son informatisation.

\section{L'ÉTAT DE LA QUESTION, AUJOURD'HUI, EN BELGIQUE}

Comme ce tour d'horizon l'a montré, le paysage belge de l'offre de produits des industries de la langue pour la traduction est très varié.

Si la TA/TAO est encore peu diffusée en Belgique, c'est qu'il n'y a qu'un seul système, METAL, dont la commercialisation débute à peine, qui permet de traiter les paires français-néerlandais : or cette combinaison représente la plus grande part (plus de la moitié) des traductions dans ce pays (exception faite des services de traduction d'organismes internationaux). Malheureusement, aucun système sur micro-ordinateur ne propose encore aucune de ces paires.

Pour d'autres combinaisons de langues, le choix est plus ouvert: c'est le cas pour les couples français-anglais et français-allemand qui sont les combinaisons les plus demandées ensuite dans la partie francophone du pays. Sur micro-ordinateur, les produits existants sur le marché belge n'offrent aujourd'hui que des paires où l'anglais est présent, dont français-anglais, et aucune paire où le néerlandais est présent.

Pour ce qui est des OAT, nombre de produits sont disponibles à des prix raisonnables. On commence à les voir apparaître chez les traducteurs pionniers, mais beaucoup de ces produits sont encore tout simplement inconnus de leurs utilisateurs potentiels.

Quant à l'intégration des OAT dans le poste de travail du traducteur (et aussi peutêtre du rédacteur multilingue), elle est... pour demain: il n'y a encore que des tentatives individuelles.

\section{DES INDUSTRIES DE LA LANGUE EN BELGIQUE?}

Si l'on peut se féliciter du haut niveau qualitatif des produits IL mis au point pour la traduction en Belgique, on doit regretter le faible nombre de produits proposés.

Il n'en existe aujourd'hui que quelques-uns. Il est par contre une activité de service qui constitue une spécialité de la Belgique : c'est la localisation de logiciels, autrement dit leur traduction et leur adaptation à des environnements culturels différents de celui d'origine. Les grands bureaux bruxellois y excellent.

De façon plus large, l'examen de INK (1992) révèle dix-sept organisations établies en Belgique et actives dans le domaine des IL, dont une douzaine sont des firmes; parmi ces organismes, quatre seulement proposent des produits et/ou services pour la traduction.

Le domaine des IL étant nouveau, et encore très peu connu, les entreprises ne sont pas nécessairement conscientes de l'éventuel potentiel ou prêtes à assumer sans garantie le risque de développements nouveaux pour un marché encore embryonnaire. Cette prudence est d'autant plus grande que l'environnement économique actuel n'est pas favorable à l'innovation, ni aux investissements. C'est particulièrement vrai dans le secteur informatique, durement touché par la récession.

La situation de la distribution des produits IL-Traduction en Belgique est plutôt satisfaisante, sauf pour les produits qui ne sont pas présents sur le marché belge : s'il est bien sûr difficile d'identifier les produits qui pourraient être utiles mais que l'on ne connaît pas, il est aussi problématique (et parfois hasardeux) de se procurer des produits non distribués en Belgique.

La présence à distance raisonnable du producteur, ou au moins de son représentant, est en effet l'un des critères qui doivent intervenir dans le choix d'un produit : la difficulté 
de l'accès au support du producteur est sans doute aussi l'un des facteurs qui freinent la diffusion en Belgique de nombreux produits IL-Traduction.

En ce qui concerne l'attitude des utilisateurs potentiels, il faut distinguer entre, d'une part, les langagiers (travailleurs de la langue), en particulier les traducteurs, et d'autre part le public (client des premiers).

Comme nous l'avons déjà dit, les traducteurs travaillent dans des cadres très différents, et souvent comme indépendants, même s'ils travaillent pour des bureaux ou des agences; ils sont par ailleurs souvent soumis à la pression de la concurrence et toujours à la tyrannie des délais.

Il résulte de ces facteurs un isolement certain qui leur fait ignorer l'existence des produits IL qui leur sont destinés. Ce manque d'information a manifestement pour effet de freiner l'expansion du marché.

Enfin, on ne peut négliger non plus l'attitude peu réceptive, sinon hostile, de certains traducteurs vis-à-vis de l'informatisation de leur travail. Le cas se présente davantage dans les administrations publiques (où, paradoxalement, on rencontre aussi les innovateurs les plus passionnés!) que dans le secteur privé. Le manque d'information, et peut-être de formation, est sans doute à nouveau l'un des facteurs qui expliquent cet état de choses.

Pour ce qui est du public professionnel, client des traducteurs, le problème se situe du côté de la formation et des attitudes : l'attention accordée à la qualité du langage, original ou traduit, est trop souvent moindre que celle accordée à la qualité de fournitures ou de productions plus matérielles. De plus, on croit encore trop qu'il suffit de connaître une langue pour traduire. En conséquence, les coûts associés aux travaux de rédaction et de traduction paraissent toujours trop élevés, et les délais trop longs. Lorsque les volumes à traduire ne sont pas trop importants (courrier, quelquefois même publicité, etc.) on en confie souvent la charge à une personne qui connaît la langue étrangère.

À cet égard, il faut noter aussi qu'en Belgique il n'est pas difficile de recruter du personnel non seulement bilingue (au sens national) mais polyglotte: c'est même l'usage dans les grandes entreprises, et plus spécialement encore dans les secteurs de haute technologie (aéronautique, espace, télécommunications, etc.) où l'on travaille très souvent en anglais. Les professionnels de ces secteurs sont donc habitués à rédiger en anglais, et la notion de traduction disparaît, sauf s'il y a des documents multilingues à produire pour l'exportation.

Par ailleurs, la mauvaise conjoncture économique de ces dernières années a imposé des économies et des restructurations qui ont souvent conduit à la disparition des services de traduction internes des grandes entreprises industrielles. Elles font plutôt appel aujourd'hui à des bureaux de traduction externes ou à des traducteurs indépendants qui n'ont pas souvent la possibilité d'investir dans des produits IL.

Dans l'important secteur des services, comme dans les administrations, on préfère encore généralement un service interne de traduction, même s'il est réduit et doit occasionnellement faire appel à des renforts extérieurs. Cela permet, en effet, de disposer en permanence d'un service prêt à traiter des traductions urgentes, d'assurer la confidentialité des documents, de maintenir un style et une terminologie maison, etc. Les technologies de la langue se diffusent dans ce secteur à des rythmes très différents: on y trouve en effet des pionniers enthousiastes, des personnes ouvertes et intéressées mais souvent mal informées, mais aussi des technophobes accrochés à leurs méthodes archaïques.

La situation en Belgique reflète donc assez exactement, à une échelle réduite, la situation générale de l'Europe (Danzin 1992).

D'un côté la circonspection des investisseurs devant un marché balbutiant entraîne une absence paralysante de moyens financiers. En même temps les politiques de R\&D nationales (ou régionales, en Belgique) n'ont pas toujours les moyens ou la volonté d'appuyer 
les efforts de recherche dans ces domaines : la CCE montre ici la voie, mais demande aussi aux gouvernements nationaux de la suivre.

De l'autre, les utilisateurs potentiels ignorent trop souvent les possibilités actuelles et futures dont ils pourraient bénéficier.

C'est, comme on voit, un cercle vicieux qui tend à se refermer sur les industries de la langue pour la traduction en Belgique, entre des producteurs souvent timorés et des utilisateurs potentiels généralement peu informés.

\section{CONCLUSION}

Les industries de la langue proposent aujourd'hui nombre de produits et de services pour la traduction, mais peu sont utilisés en Belgique francophone, et moins encore l'ont été ou y sont développés, à de très remarquables exceptions près.

Divers facteurs peuvent expliquer cette situation: la structure de l'économie nationale et régionale, la conjoncture actuelle, l'instabilité des efforts de recherche, l'absence de systèmes traitant les paires de langues nationales sur micro-ordinateur, et, surtout, le manque d'information des utilisateurs potentiels.

Malgré les besoins, cet état de choses en forme de cercle vicieux ne stimule évidemment pas l'utilisation des produits et des services IL-Traduction, et pas davantage leur commercialisation, ni encore moins leur développement local.

L'information des utilisateurs est sans doute le premier moyen d'action: la méconnaissance des produits par leurs utilisateurs potentiels bloque en effet leur diffusion mais décourage aussi leurs producteurs et distributeurs.

Il est donc souhaitable de favoriser et d'activer la circulation de l'information, notamment entre les différents observatoires du réseau créé par l'Agence de coopération culturelle et technique des pays francophones (ACCT) d'une part, et de l'autre entre les Observatoires et les acteurs concernés dans leurs régions respectives.

Les professionnels de la traduction devrait pouvoir recevoir, pendant ou après leur études, une formation adaptée à l'émergence des nouvelles technologies dans le secteur.

Il faut encore encourager les activités de R\&D dans le secteur et favoriser particulièrement les synergies entre les centres de recherche et les industriels du domaine dans un esprit d'abord plus collaboratif que compétitif, par exemple, en s'efforçant de produire des ressources (lexiques, grammaires, etc) réutilisables et en les mettant dans le domaine public, de façon à amorcer et à permettre le prolongement de ces développements technologiques.

Des mesures d'encouragement à l'égard des producteurs et distributeurs de produits IL pour la traduction, et peut-être des utilisateurs potentiels, apporteraient sans doute une impulsion supplémentaire.

Enfin, souhaitons que la suppression des barrières douanières en Europe, laquelle n'entrânera pas pour autant la disparition des barrières linguistiques, constitue une stimulation pour les développeurs et distributeurs, et aussi une occasion de sensibiliser le public à l'importance de la qualité du langage en général, et au rôle de la traduction en particulier.

\section{RÉFÉRENCES}

BENETT, W. S. (1991) (compilateur): A General Overview of the METAL Machine Translation System, Siemens-Nixdorf, première version.

Commission des Communautés européennes : communications personnelles et documents divers SYSTRAN.

CLAS, A. et H. SAFAR (Éds) (1992) : L'environnement traductionnel. La station de travail du traducteur de l'an 2001, Actes du Colloque AUPELF, Mons 1991, Presses de 1'Université de Québec.

COLING 92 (1992): Proceedings of the fifteenth International Conference on Computational Linguistics, Nantes, 23-28/8/92, ICCL. 
COPETTE, A. et J. M. VANDE WALLE (1993) : Traduction/Interprétation Formation - Emploi : l'avenir, Actes du colloque ATIM, Mons, Presses Universitaires de Mons.

CORBEIL, J.-C. (1990): Les industries de la langue : un domaine à la recherche de lui-même, Gouvernement du Québec, Conseil de la langue française, Québec.

DANZIN, A. et al. (1992): Vers une infrastructure linguistique européenne, rapport à la Commission des Communautés européennes, DG XIII, CEE, Bruxelles.

GUÉRAIN, S. (1990) : Vous avez dit Traducteur? Enquête sur le Traducteur en Belgique, UMH, École d'Interprètes Internationaux, Mons.

HUTCHINS, W. J. et H. SOMERS (1992) : An Introduction to Machine Translation, London, Academic Press.

HUTCHINS, W. J. (1986) : Machine Translation: Past, Present, Future, Ellis Horwood Series in Computers and their Applications, Chichester, Ellis Horwood, John Wiley \& Sons.

INK (1992): INK Luxembourg SA, The 1992 Language Engineering Directory, Language Industries Survey, Commission of the European Communities, Luxemburg.

JEIDA (1992): The Survey of the Current Status of Research and Future Trends in Machine Translation and Natural Language Processing, JEIDA, Tokyo.

KAY, M. (1980): The Proper Place of Men and Machines in Machine Translation, Research Report CSL-80-11, Xerox PARC, Palo Alto, Cal.

KING, M. (1992) : «L'évaluation des systèmes de traduction automatique dans le cadre d'un service de traduction», Meta, 37-4, Montréal, Presses de l'Université de Montreál.

MACKLOVITCH, E. (1992): «PTT-2: la deuxième version du poste de travail du traducteur du CCRIT», A. Clas et $\mathrm{H}$. Safar, L'environnement traductionnel. La station de travail du traducteur de l'an 2001, Actes du Colloque AUPELF, Mons 1991, Presses de l'Université du Québec.

MACKLOVITCH, E. (1992): The Trial of the Translator's Workstation at the Canadian Translation Bureau Secretary of State of Canada Translation Bureau.

MELBY, A. K. (1992) : «Pour le traducteur : un poste de travail à trois niveaux d'assistance», A. Clas et H. Safar, L'environnement traductionnel. La station de travail du traducteur de l'an 2001, Actes du Colloque AUPELF, Mons 1991, Presses de l'Université du Québec.

MELBY, A. K. (1982): «Multi-level Translation Aids in a Distributed System», Proceedings of COLING'82, pp. 215-220, Prague.

META (1992): Numéro spécial Études et recherches en traductique, sous la direction de Monique C. Cormier et Dominique Estival, vol. 37 n $^{\circ}$ 4, décembre 1992, Montréal, Presses de l'Université de Montréal.

NAGAO, M. (1984): «A Framework of a Mechanical Translation between Japanese and English by Analogy Principle», A. Elithorn et R. Banerji (Éds.), Artificial and Human Intelligence, Amsterdam, North-Holland, pp. $173-180$.

NIRENBURG, S., CARBONELL, J., TOMITA, M. et K. GOODMAN (1992) : Machine Translation a Knowledge-based Approach, Morgan Kaufmann Publishers, San Mateo.

OCIL et OWIL (1991): Connaissez-vous les industries de la langue? Perspectives des années 1990-2000, Agence de coopération culturelle et technique, Sommet francophone de Chaillot, Paris.

REICHLING, A. (1992) : «EURODICAUTOM, ou la terminologie en l'an 2001», A. Clas et H. Safar, L'environnement traductionnel. La station de travail du traducteur de l'an 2001, Actes du Colloque AUPELF, Mons 1991, Presses de l'Université du Québec.

SCHNEIDER, Th. (1992) : «User Driven Development: METAL as an Integrated Multilingual System», Meta, 37-4, Montréal, Presses de l'Université de Montréal.

Siemens-Nixdorf Informationssysteme, Documents divers sur METAL.

TIL4-5-6 (1991): «La tribune des industries de la langue», Ingéniérie linguistique: problématiques 1995, numéro 4-5-6 spécial SIL, 11/91, OFIL, Paris.

TMI (1992): Fourth International Conference on Theoretical and Methodological Issues in Machine Translation, Empiricist vs Rationalist Methods, Proceedings of the Conference CCRIT-CWARC, Montréal

TREMBLAY, K. (1992) : «La technologie au service du traducteur», A. Clas et $\mathrm{H}$. Safar, L'environnement traductionnel. La station de travail du traducteur de l'an 2001, Actes du Colloque AUPELF, Mons 1991, Presses de l'Université du Québec.

VAN STEENBERGHE, T. (1992) : Les industries de la langue secteur traduction, rapport pour la Région wallonne de Belgique, UCL.

VARILE, G. B. et A. ZAMPOLLI (1992): Synopses of American, European and Japanese Projects presented at the International Projects Day, at COLING-92, Nantes, juillet 1992, Linguistica Computazionale, VIII, Pisa. 


\section{ANNEXE}

- Instituts de recherche et établissement d'enseignement

Instituts de recherche - Région wallonne et Bruxelles

CSL Centre Software de Liège

UCL Université Catholique de Louvain à Louvain-la-Neuve

ULB Université Libre de Bruxelles

ULg Université de Liège

UMH Université de Mons-Hainaut

Établissements d'enseignement - Région wallonne et Bruxelles

Études principales complètes :

École d'Interprètes Intemationaux (EII), Mons

Institut Cooremans, Bruxelles

Institut Libre Marie Haps (ILMH), Bruxelles

Institut Supérieur de Traducteurs et Interprètes (ISTI), Bruxelles

Études partielles ou de complément :

FUNDP, Facultés universitaires N-D de la Paix, Namur

FUSL, Facultés universitaires Saint-Louis, Bruxelles

UCL, Université catholique de Louvain à Louvain-la-Neuve

ULB, Université libre de Bruxelles

ULg, Université de Liège

UMH, Université de Mons-Hainaut

\section{ACRONYMES}

ACCT Agence de coopération culturelle et technique (des pays francophones)

CCE Commission des Communautés européennes

CCRIT Centre canadien de recherche sur l'informatisation du travail

CD-ROM Compact Disc - Read Only Memory

EBMT Example Based Machine Translation

FAHQT Full Automatic High Quality Translation

IL Industries de la langue

IRSIA Institut pour la recherche scientifique, industrielle, et agronomique

MBMT Memory Based Machine Translation

MT Machine Translation

OAT Outils d'aide à la traduction

OCR Optical Character Recognition

OWIL Observatoire wallon des industries de la langue

PAO Publication assistée par ordinateur

R\&D Recherche et développement

RBMT Rule Based Machine Translation

TA Traduction automatique

TAO Traduction assistée par ordinateur

TEI Text Encoding Initiative

THAO Traduction humaine assistée par ordinateur 